Int. J. Dev. Biol. 65: 563-570 (2021)

https://doi.org/10.1387/ijdb.210066rs

\title{
Expression of two uncharacterized protein coding genes in zebrafish lateral line system
}

\author{
SANA FATMA ${ }^{1,2}$ RAVINDRA KUMAR ${ }^{3}$, ANSHUMAN DIXIT ${ }^{1}$ and RAJEEB K. SWAIN ${ }^{*, 1}$ \\ ${ }^{1}$ Institute of Life Sciences, Nalco Square, Chandrasekharpur, Bhubaneswar, ${ }^{2}$ Manipal Academy of Higher Education, \\ Manipal, Karnataka and ${ }^{3}$ School of Biotechnology, National Institute of Technology Calicut, Kerala, India
}

\begin{abstract}
The lateral line system is a mechanosensory organ of fish and amphibians that detects changes in water flow and is formed by the coordinated action of many signalling pathways. These signalling pathways can easily be targeted in zebrafish using pharmacological inhibitors to decipher their role in lateral line system development at cellular and molecular level. We have identified two uncharacterized proteins, whose mRNA are expressed in the lateral line system of zebrafish. One of these proteins, uncharacterized protein LOC564095 precursor, is conserved across vertebrates and its mRNA is expressed in posterior lateral line primordium (pLLP). The other uncharacterized protein, LOC100536887, is present only in the teleost fishes and its mRNA is expressed in neuromasts. We show that inhibition of retinoic acid (RA) signalling reduces the expression of both of these uncharacterized genes. It is reported that inhibition of RA signalling during gastrulation starting at 7 hours post fertilization (hpf) abrogates pLLP formation, and inhibition of RA signalling at 10 hpf delays the initiation of pLLP migration. Here, we show that inhibition of RA signalling before and during segmentation (9-16 hpf) results in delayed initiation and reduced speed of pLLP migration, as well as inhibition of posterior neuromasts formation.
\end{abstract}

KEY WORDS: Lateral line system, posterior lateral line primordium, neuromasts, uncharacterized proteins

\section{Introduction}

The lateral line is a mechanosensory system that is present in fishes and most amphibians in order to detect the pattern of water movement and to spot the location of prey and predators (Coombs and Van Netten, 2005). Its functional units are neuromasts (Kornblum et al., 1990; Bleckmann and Zelick, 2009). The lateral line system in zebrafish is divided into two parts: the anterior lateral line (aLL), present around the head; and the posterior lateral line ( $p L L)$, which runs in the trunk and the tail. These are derived from anterior and posterior lateral line placodes, respectively (Andermann et al., 2002; Ghysen and Dambly-Chaudiere, 2004). The pLL is generated by the deposition of neuromasts by a migrating primordium (pLLP) during development. The migratory pLLP consists of around 140 cells, which emerge behind the otic placode at around 20-22 hpf and begin to migrate towards the tip of the tail, which is completed by 42 hpf (Gompel et al., 2001; Dalle Nogare and Chitnis, 2017). During this migration, they deposit cells as proto-neuromasts at regular intervals throughout the horizontal myoseptum to generate 5 neuromasts (L1-L5) in the trunk region and 2-3 terminal neuromasts at the tip of the tail (Gompel et al., 2001). These neuromasts undergo a process of morphogenesis, become epithelized and mature into rosettes with sensory hairs at their center (Lecaudey et al., 2008; Hava et al., 2009). Approximately 20 cells of the original placode remain to form neurons of lateral line ganglion which later follow migratory path of pLLP and establish connections with sensory hair cells (Metcalfe, 1985; Chitnis et al., 2012). The migratory pLLP has a dynamic structure that varies from the leading end to the trailing end. Leading cells are mesenchymal and flatter in appearance, while cells at the trailing end are epithelial and have columnar morphology called proto-neuromasts, which later develop into rosettes (Lecaudey et al., 2008). More cells are added to the leading end of migratory pLLP by division of existing cells that in turn shifts the newly formed neuromasts more towards the trailing end (Nechiporuk and Raible, 2008; Chitnis et al., 2012).

Abbreviations used in this paper: aLL, anterior lateral line; DEAB, N,N-diethylaminobenzaldehyde; DMSO, dimethyl sulfoxide; pLL, posterior lateral line; pLLP, posterior lateral line primordium; RA, Retinoic acid.

\footnotetext{
*Address correspondence to: Rajeeb K. Swain. Institute of Life Sciences, Nalco Square, Chandrasekharpur, Bhubaneswar - 751023, India. Tel: +916742304301. E-mail: rkswain@ils.res.in - iD https://orcid.org/0000-0003-4815-8221
} 
Several signalling pathways regulate the process of lateral line placode formation, primordium migration, proto-neuromast formation and maturation in zebrafish. Wnt, Fgf and Bmp signalling control the expansion of posterior lateral line placode (Nikaido et al., 2017). Wnt-dependent Fgf signalling is responsible for protoneuromast formation (Aman and Piotrowski, 2008). Fgf signalling also triggers proto-neuromast maturation, formation of sensory hair cells within the neuromasts, and induction of anterior lateral line placode (Nikaido et al., 2017; Lecaudey et al., 2008; Nechiporuk and Raible, 2008). Chemokine signals such as chemokine receptors (cxcr4 and cxcr7) and their ligand sdf1a regulate pLLP migration throughout the horizontal myoseptum (David et al., 2002; Haas and Gilmour, 2006; Dambly-Chaudière et al., 2007). Notch signalling regulates the development of sensory hair cell progenitors present in the neuromasts (Matsuda and Chitnis, 2010). Knockdown of $\operatorname{tg} f \beta 1$ a reduced neuromast numbers with increased inter-neuromast distance, thus highlighting the role of TGF $\beta$ signalling in pLL development (Xing et al., 2015). It is reported that the RA signalling pathway regulates posterior lateral line placode formation during late gastrulation stage from 8 to $10 \mathrm{hpf}$ (Sarrazin et al., 2010). Inhibition of RA synthesis from 10 to $24 \mathrm{hpf}$ delays initiation of pLLP migration (Nikaido et al., 2017).

Whole-genome sequencing along with advancements of bioinformatics tools have identified many predicted open reading frames (ORFs) (Brent, 2005). When the proteins encoded by these ORF do not show significant homology with the proteins of known function, they are called uncharacterized, unknown, hypothetical, or predicted proteins (henceforth, uncharacterized) (ljaq et al., 2015). These uncharacterized genes comprise a significant fraction of the genome of most organisms. However, assigning functions to these predicted ORFs is challenging (Hawkins and Kihara, 2007; ljaq et al., 2015). Here, we have identified two uncharacterized proteins, and show that the mRNA of "uncharacterized protein LOC564095 precursor" and "uncharacterized protein LOC100536887" are expressed in zebrafish lateral line system. We show that RA signalling regulates their expression, and hypothesize that the effect of RAon pLLP migration and neuromast development in zebrafish embryos may be partly regulated through these proteins.

\section{A}

Unconserved 12345678910 Conserved
C

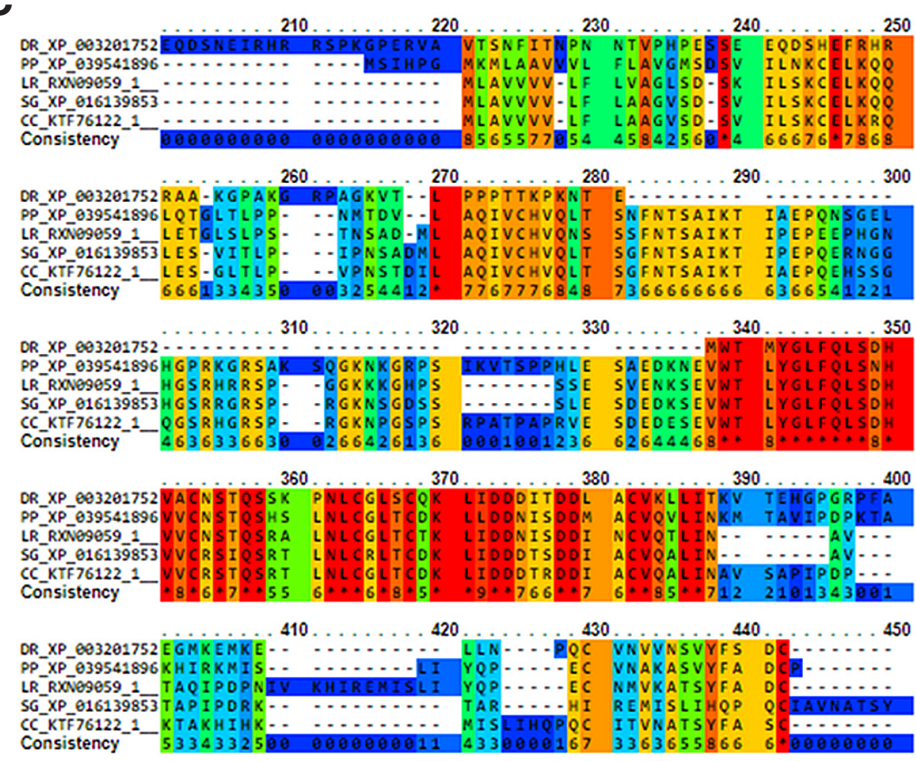

B

\begin{tabular}{|c|c|c|c|}
\hline & $\begin{array}{c}\text { DR } \\
\text { NP_001038506.1 }\end{array}$ & $\begin{array}{c}\text { MIVI } \\
\text { NP_705733.2 }\end{array}$ & $\begin{array}{c}\text { HS } \\
\text { NP_001287968.1 }\end{array}$ \\
\hline $\begin{array}{c}\text { DR } \\
\text { NP_001038506.1 }\end{array}$ & 100.00 & 32.35 & 35.71 \\
\hline $\begin{array}{c}\text { MIM } \\
\text { NP_705733.2 }\end{array}$ & 32.35 & 100.00 & 63.85 \\
\hline $\begin{array}{c}\text { HS } \\
\text { NP_001287968.1 }\end{array}$ & 35.71 & 63.85 & 100.00 \\
\hline
\end{tabular}

D

\begin{tabular}{|c|c|c|c|c|c|}
\hline & $\begin{array}{c}\text { DR } \\
\text { XP_003201752.1 }\end{array}$ & $\begin{array}{c}\text { PP } \\
\text { XP_039541896.1 }\end{array}$ & $\begin{array}{c}\text { LR } \\
\text { RXN09059.1 }\end{array}$ & $\begin{array}{c}\text { SG } \\
\text { XP_016139853.1 }\end{array}$ & $\begin{array}{c}\text { CC } \\
\text { KTF76122.1 }\end{array}$ \\
\hline $\begin{array}{c}\text { DR } \\
\text { XP_003201752.1 }\end{array}$ & 100.00 & 54.90 & 52.11 & 53.16 & 51.53 \\
\hline $\begin{array}{c}\text { PP } \\
\text { XP 039541896.1 }\end{array}$ & 54.90 & 100.00 & 71.73 & 72.25 & 71.72 \\
\hline $\begin{array}{c}\text { LR } \\
\text { RXN09059.1 }\end{array}$ & 52.11 & 71.73 & 100.00 & 76.04 & 73.44 \\
\hline $\begin{array}{c}\text { SG } \\
\text { XP 016139853.1 }\end{array}$ & 53.16 & 72.25 & 76.04 & 100.00 & 85.42 \\
\hline $\begin{array}{c}\text { CC } \\
\text { KTF76122.1 }\end{array}$ & 51.53 & 71.72 & 73.44 & 85.42 & 100.00 \\
\hline
\end{tabular}

Fig. 1. Homologs of uncharacterized protein LOC564095 precursor and uncharacterized protein LOC100536887 in other species. (A,B) The DUF4634 domain was taken for BLASTP search to identify the proteins containing this domain in other species. Multiple sequence alignment and percentage identity matrix of DUF4634 domain present in uncharacterized protein LOC564095 precursor (NP_001038506.1) of zebrafish with the domain from homologous proteins in human (NP_001287968.1) and mice (NP_705733.2). (C,D) Multiple sequence alignment and percentage identity matrix of uncharacterized protein LOC100536887 (XP_003201752.1) with its homologs in other species. LOC100536887 homologs are present only in the class Teleostei. Sequences were aligned using PRALINE, percentage identity matrix were generated using MUSCLE. DR, Danio rerio; MM, Mus musculus; HS, Homo sapiens; PP, Pimephales promelas; LR, Labeo rohita; SG, Sinocyclocheilus graham; CC, Cyprinus carpio. 

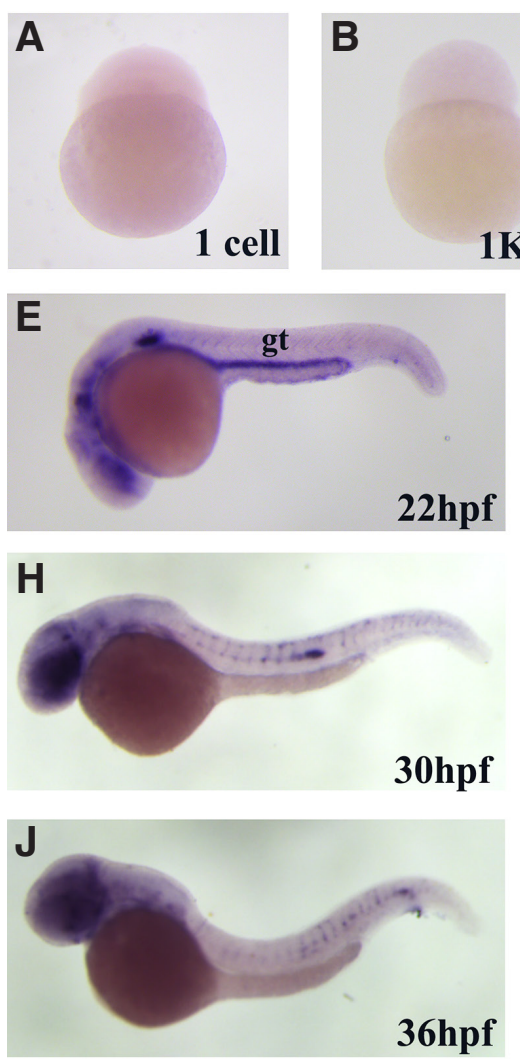

B
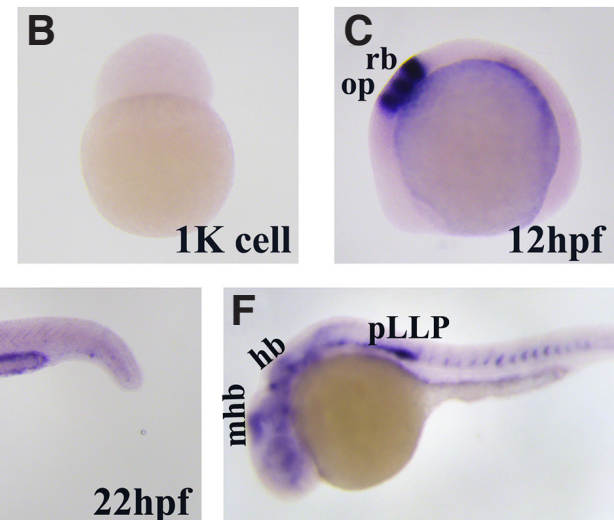
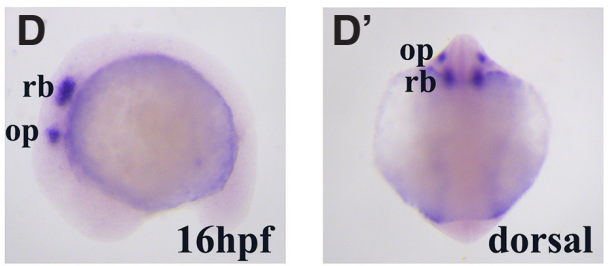

$24 \mathrm{hpf}$
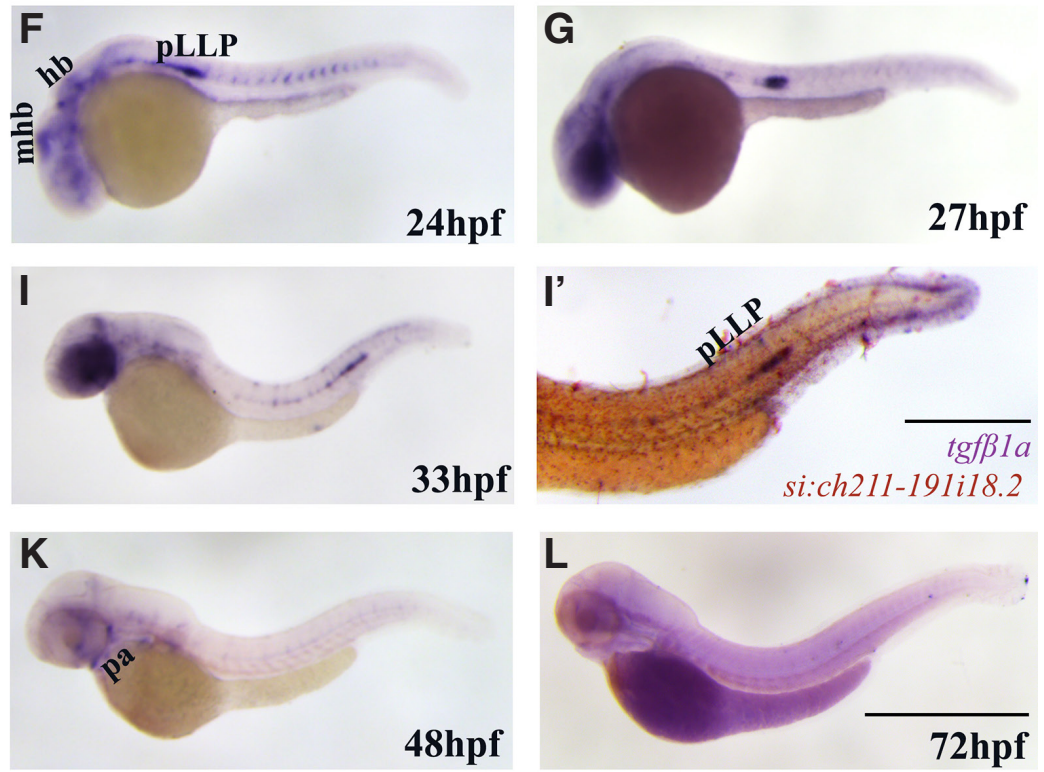

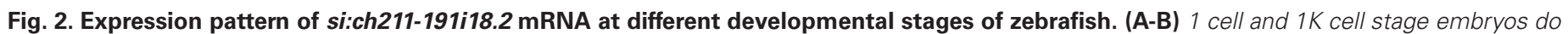

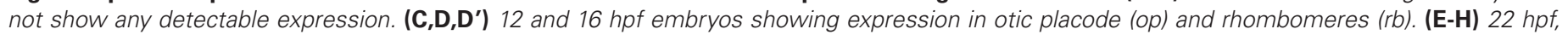

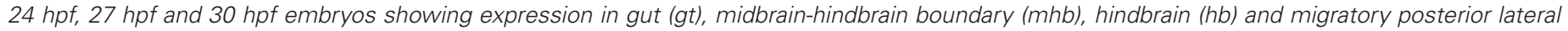

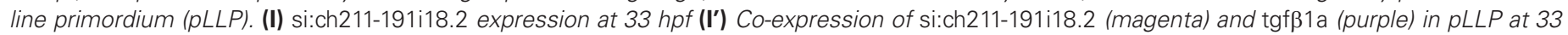

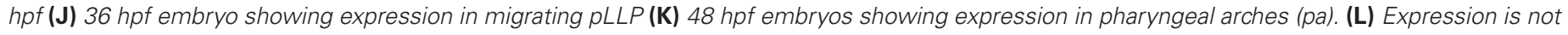
detectable in 72 hpf embryos. Scale bar $500 \mu \mathrm{m}$ for all images except I' for which the scale bar is $250 \mu \mathrm{m}$.

\section{Results}

\section{Uncharacterized protein LOC564095 precursor is conserved and uncharacterized protein LOC100536887 is a lineage- specific protein}

Zebrafish uncharacterized protein LOC564095 precursor (NP_001038506.1) is a 124 amino acid long protein with a DUF4634 (Domain of Unknown Function 4634) domain. The DUF4634 domain comprises 83-98 percent of the total protein sequence where it is present. Multiple sequence analysis of DUF4634 domain, present in uncharacterized protein LOC564095 precursor with its homolog in mice (NP_705733.2) and humans (NP_001287968.1), showed $32.35 \%$ and $35.71 \%$ sequence identity respectively (Fig. 1 A,B). The identity between mice and humans was $63.85 \%$.

Zebrafish uncharacterized protein LOC100536887 (XP_003201752.1) is a 367 amino acid long protein and has a DUF4106 domain, a lysozyme-like domain and a calcium-binding site. The BLAST analysis of this protein revealed that it is conserved among bony fishes belonging to the class Teleostei, suggesting that it is a lineage-specific protein (Fig. 1 C,D) (Lespinet, 2002). The DUF4106 domain is also present in Hypothetical Protein TVAG_358080 of protozoan parasite Trichomonas vaginalis, which does not contain the lysozyme-like domain, and calcium-binding site present in uncharacterized protein LOC100536887.

\section{The mRNA of uncharacterized protein LOC564095 precursor is expressed in pLLP}

Zebrafish uncharacterized protein LOC564095 precursor (NP_001038506.1) is encoded by si:ch211-191i18.2 gene. It has two predicted transcripts, ENSDART00000150919.3 and ENSDART00000151034.2. We carried out whole-mount in situ hybridization (WISH) at different developmental stages of zebrafish to find out the temporal and spatial expression pattern of si:ch211-191i18.2 mRNA. The antisense probe was designed to recognize both the splice variants. Its mRNA expression was not visible in the early embryonic stages (Fig. 2 A,B). At 12 and $16 \mathrm{hpf}, \mathrm{mRNA}$ expression was seen in the otic placode and rhombomeres (Fig. 2 C,D,D'). In 22 hpf embryos, expression was observed in the hindbrain, midbrain-hindbrain boundary, gut, and migratory pLLP, and was also seen in the later stages of development (Fig. 2E). The expression domain of si:ch211-191i18.2 gene in migratory primordium continued to move behind the otic placode towards the tip of the tail as the development progressed (Fig. 2 E-J). Double in situ hybridization with $\operatorname{tg} \beta \beta 1 a$ (a lateral line primordium marker) (Xing et al., 2015) confirmed si:ch211-191i18.2 expression in the pLLP (Fig. 2l'). In 48 hpf embryos, expression was observed in the pharyngeal arches (Fig. $2 \mathrm{~K}$ ), but no detectable expression was observed in the $72 \mathrm{hpf}$ embryos (Fig. 2L). 

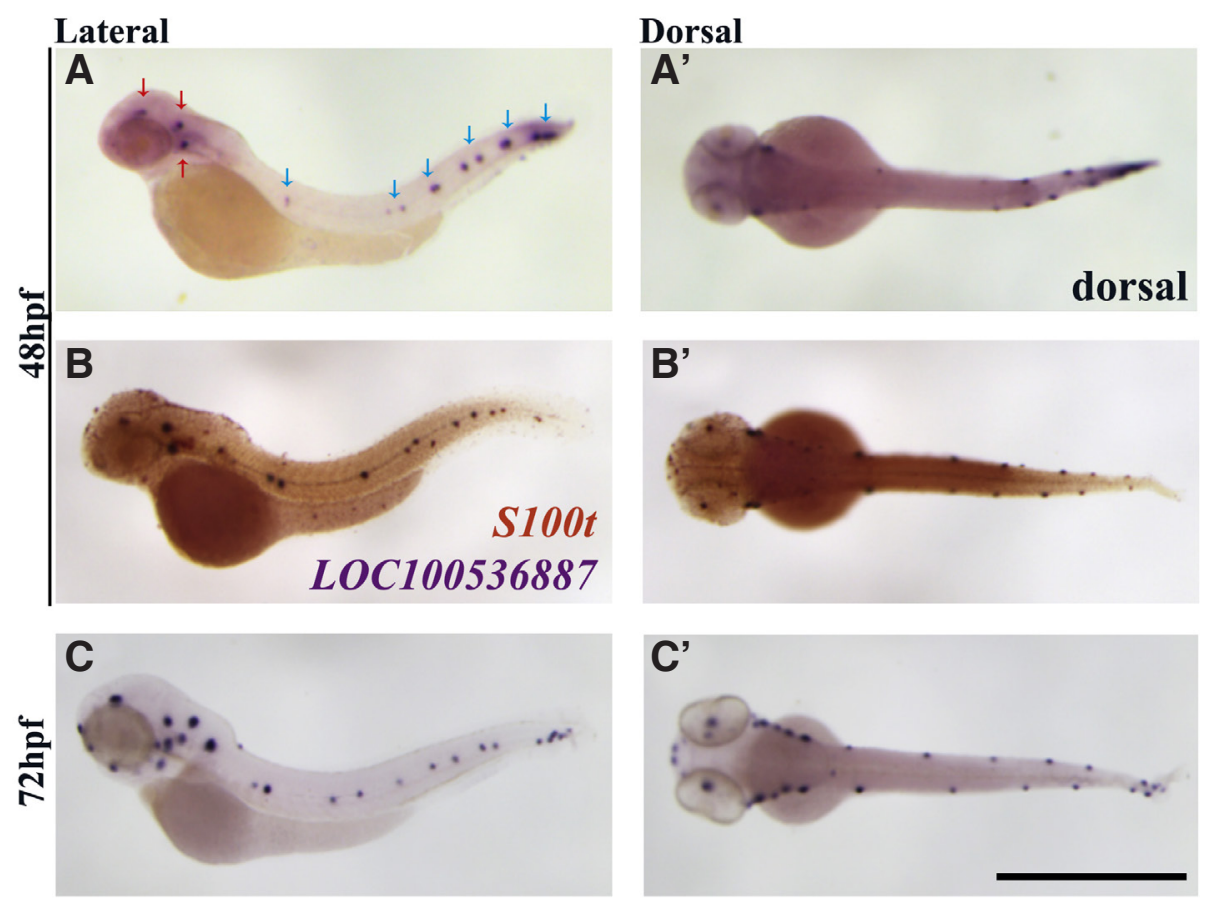

Fig. 3. Expression pattern of LOC100536887 at different developmental stages of zebrafish embryos. (A, $\left.\mathbf{A}^{\prime}\right) 48$ hpf embryos showing expression in anterior (red arrow) and posterior (blue arrow) neuromasts. (B, $\left.\mathbf{B}^{\prime}\right)$ Co-expression of LOC100536887 (purple) and S100t (magenta) in neuromasts at 48 hpf embryo. (C,C') 72 hpf embryos showing expression in anterior and posterior neuromasts. Scale bar $500 \mu \mathrm{m}$.

(Kraemer et al., 2008) confirmed the location of LOC100536887 in neuromasts (Fig. 3B).

\section{Inhibition of Retinoic acid signalling re- duces the expression ofsi:ch211-191i18.2 and LOC100536887}

$R A$ signalling is required during late gastrulation for posterior lateral line development (Sarrazin et al., 2010; Nikaido et al., 2017). We checked whether RA has any effect on si:ch211-191i18.2 (codes uncharacterized protein LOC564095 precursor) and LOC100536887 (codes uncharacterized protein LOC100536887) expression. We treated the embryos with either $10 \mu \mathrm{M} \mathrm{N}, \mathrm{N}-$ The mRNA of uncharacterized protein LOC100536887 is expressed in lateral line neuromasts

Uncharacterized protein LOC100536887 (XP_003201752.1) is encoded by LOC100536887 gene. It has one known transcript XM_003201704.5. An antisense probe was designed to check the expression pattern of LOC100536887gene at different developmental stages of zebrafish embryos. Its expression was not detectable in embryos from 1 cell to $36 \mathrm{hpf}$ (data not shown). At $48 \mathrm{hpf}$, its expression was observed both in anterior and posterior lateral line neuromasts, and was also seen in 72 hpf embryos (Fig. 3A and C). Double in situ hybridization with S100t (a neuromasts marker)

A

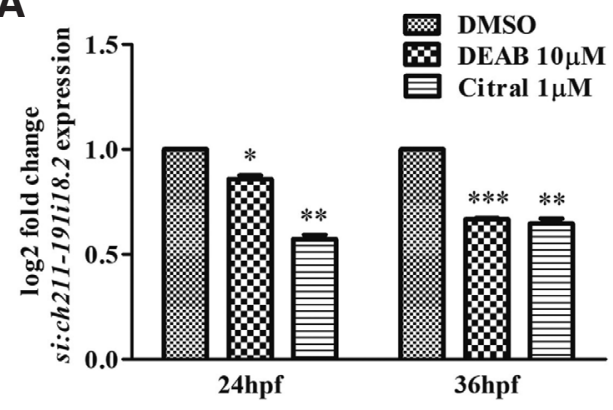

C

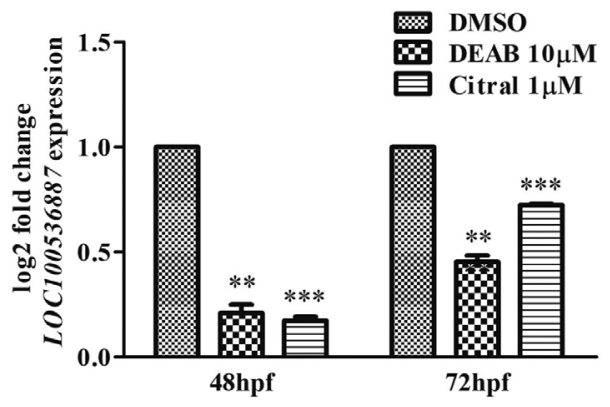

B

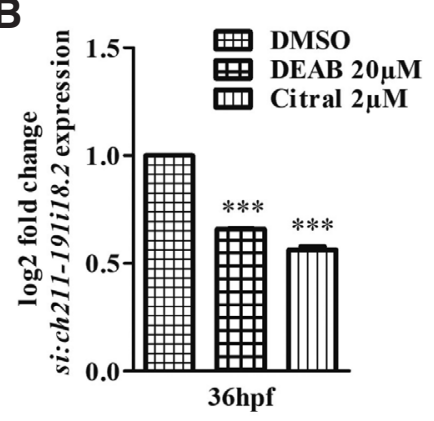

D

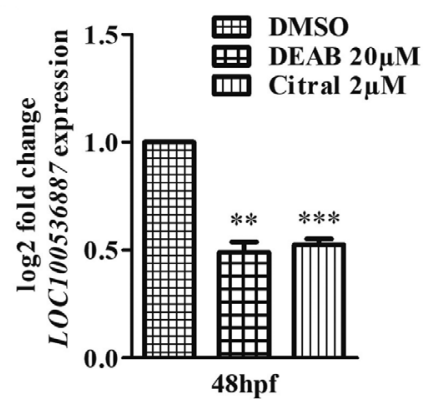

diethylaminobenzaldehyde (DEAB), an inhibitor of retinaldehyde dehydrogenase; $1 \mu \mathrm{M}$ citral (that inhibits the oxidation of retinol to $\mathrm{RA}$ ); or vehicle control dimethyl sulfoxide (DMSO). Early inhibition of RA from 9 to $16 \mathrm{hpf}$ significantly reduced the expression of si:ch211-191i18.2 gene at 24 and $36 \mathrm{hpf}$ (Fig. 4A). It is a possible that the decreased expression of si:ch211-191i18.2 may be due to delayed development of lateral line system upon RA inhibition. To rule out this possibility, we treated the embryos with $D E A B$ or citral from $24 \mathrm{hpf}$ at which point the primordium migration has already started. The expression of si:ch211-191i18.2 was checked at 36 hpf after treating the embryos with RA inhibitors from 24 to $36 \mathrm{hpf}$. The RT PCR analysis showed that with $20 \mu \mathrm{M} \mathrm{DEAB}$ and $2 \mu \mathrm{M}$ citral, there was a significant reduction of si:ch211-191i18.2 expression at $36 \mathrm{hpf}$ (Fig. 4B).

As LOC100536887 gene is expressed $48 \mathrm{hpf}$ onwards (Fig. 3), its expression upon RA signalling inhibition was checked at $48 \mathrm{hpf}$ and $72 \mathrm{hpf}$. Early inhibition of RA between 9 to $16 \mathrm{hpf}$ reduced the expression of LOC100536887 in DEAB or citral treated embryos (Fig. 4C). The expression of LOC100536887 was checked further by treating the embryos with $20 \mu \mathrm{M}$ DEAB and $2 \mu \mathrm{M}$ citral from 24 to 48 hpf. The reduced expression of LOC100536887 transcript was found at $48 \mathrm{hpf}$ upon RA signalling inhibition (Fig. 4D).

Fig. 4. Effect of DEAB and citral on si:ch211-191i18.2 and LOC100536887 gene expression. RT-PCR analysis of si:ch211-191i18.2 transcript of DEAB, citral or DMSO treated embryos (A) at 24 and $36 \mathrm{hpf}$, drug treatment time 9 to $16 \mathrm{hpf}$ (B) at $36 \mathrm{hpf}$, drug treatment time 24 to $36 \mathrm{hpf}$. RT-PCRanalysis of $L O C 100536887$ transcript of DEAB, citral or DMSO treated embryos (C) at 48 and 72 hpf, drug treatment time 9 to $16 \mathrm{hpf}$ (D) at $48 \mathrm{hpf}$, drug treatment time 24 to 48 hpf. $n=4$, mean $\pm S E M$, paired t test, two tailed; ${ }^{*} p$-value $<0.05,{ }^{* *} p$-value $<0.01$ and ${ }^{* *} p$-value $<0.001$. 
A

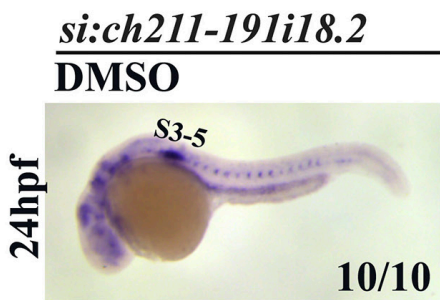

\section{DEAB}
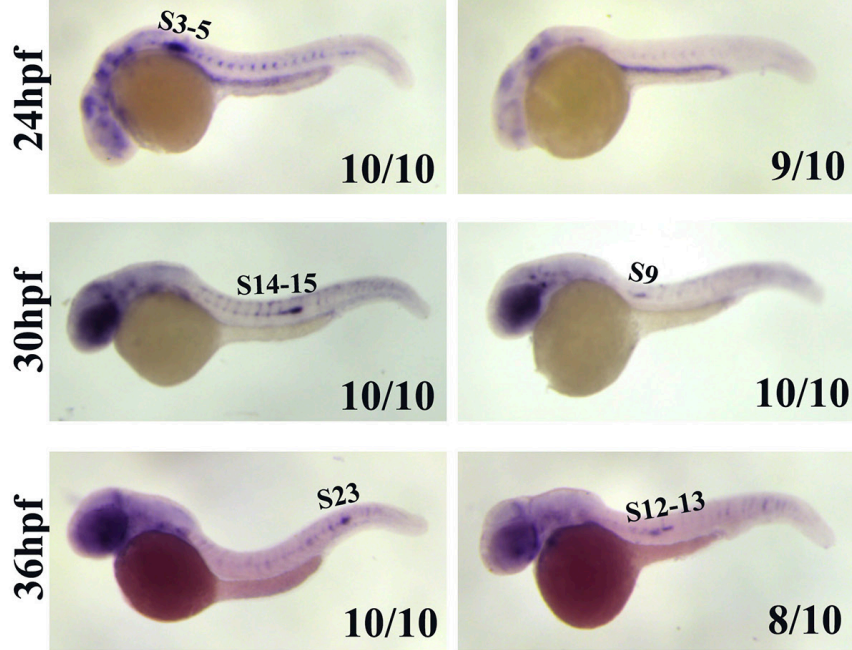

C

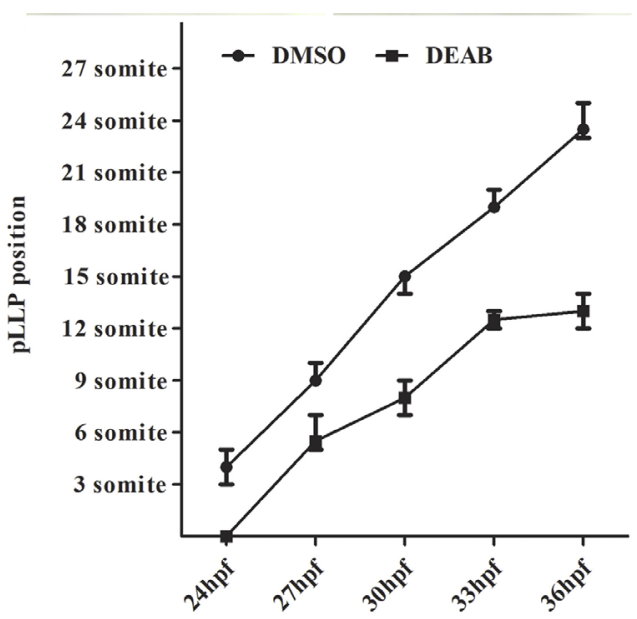

Fig. 5. Effect of DEAB on lateral line primordium migration. Initiation of pLLP migration was delayed and the speed of migration was slowed-down in DEAB treated embryos compared with DMSO treated control embryos. Observations were done at $24 \mathrm{hpf}, 30 \mathrm{hpf}$ and $36 \mathrm{hpf}$ using (A) si:ch211-191i18.2 and (B) tgf $\beta 1$ a transcripts as pLLP markers. "S" represents the somite position where $\mathrm{pLLP}$ had reached. Scale bar $500 \mu \mathrm{m}$. Ratios at right corner of each image indicate number of embryos showing the pattern of expression. (C) The speed of pLLP migration as measured by its position with respect to somite number at mentioned time points (hpf) in DEAB and DMSO treated embryos between 24 to $36 \mathrm{hpf}(n=6)$.

\section{Inhibition of retinoic acid signalling results in delayed initia- tion and reduced speed of pLLP migration}

It is reported that inhibition of RA signalling by DEAB treatment from 7 to $24 \mathrm{hpf}$ completely inhibits pLLP formation. DEAB treatment from 10 to $24 \mathrm{hpf}$ does not alter pLLP formation but delays primordium migration, and treatment from 14 to $24 \mathrm{hpf}$ had no effect on pLLP formation or migration (Nikaido et al., 2017). Two uncharacterized genes reported here (si:ch211-191i18.2 and LOC100536887) are expressed in pLLP and neuromast respectively. Both are down regulated upon RA signalling inhibition. We wanted to know whether inhibition of RAsignalling affects only pLLP
B
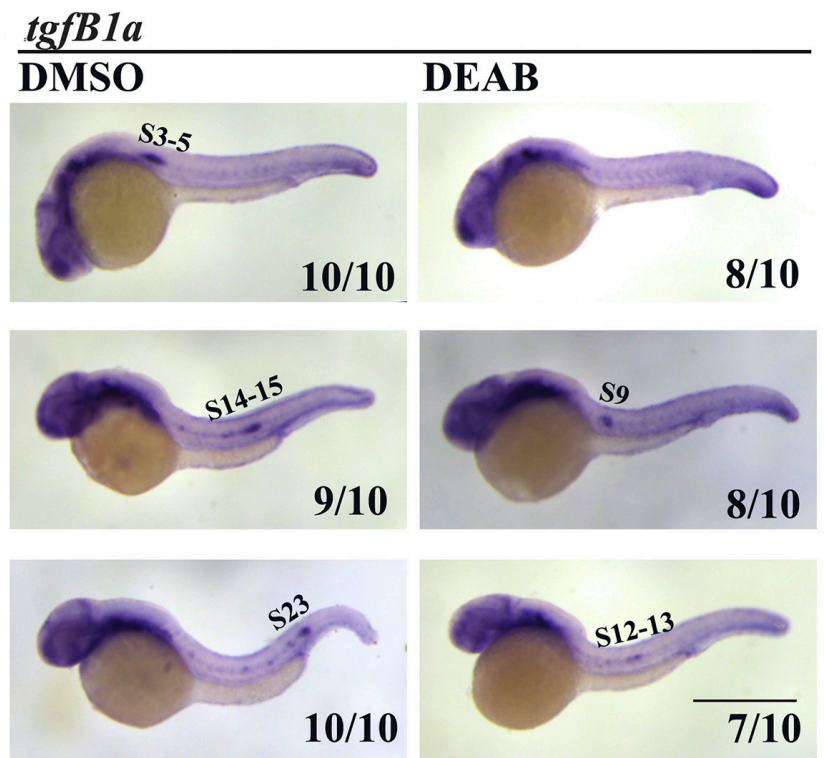

migration initiation, or also the speed at which they migrate. To address this question, embryos were treated with DEAB or DMSO from 9 to $16 \mathrm{hpf}$ and its effect on pLLP migration was checked by WISH using expression of si:ch211-191i18.2 and tgf $\beta 1$ a genes at 24,30 and 36 hpf. pLLP had migrated to the position of 3-5 somite in DMSO treated $24 \mathrm{hpf}$ embryos as detected by expression of si:ch211-191i18.2 and tgf $\beta 1 a$. pLLP migration was not initiated by this time in DEAB treated embryos. In 30 hpf DEAB treated embryos, si:ch211-191i18.2 and tgf $\beta 1$ a genes were detected in pLLP at somite position 9, whereas the pLLP had migrated to somite position 14 to 15 in DMSO treated embryos. Similarly, pLLP was observed at somite position 12 to 13 in DEAB treated embryos, while it had reached the tail in DMSO treated embryos (Fig. 5A and $B)$. To check the speed of migratory pLLP, a comparative analysis was performed between 24 to $36 \mathrm{hpf}$ in DEAB and control DMSO treated embryos with three hours' time interval. It was observed that in DMSO treated embryos, the migration of pLLP is almost uniform, with an average speed of 1.6 somite length per hour. The pLLP migration was slow in DEAB treated embryos and moved at an average speed of 1 somite per hour. Moreover, the speed of pLLP migration in DEAB treated embryos was not uniform, as no change in pLLP position was observed between 33 to $36 \mathrm{hpf}$ (Fig. $5 \mathrm{C})$. These results suggest that RA inhibition not only abrogates or delays initiation of pLLP migration as reported earlier (Nikaido et al., 2017), but also affects the speed of primordium migration in zebrafish embryos.

\section{Inhibition of retinoic acid signalling affects neuromast deposi- tion throughout the body}

The primary posterior lateral line system is fully developed by $3 \mathrm{dpf}$ (Nuñez et al., 2009). It is reported that treatment with DEAB between 8 to $10 \mathrm{hpf}$ reduced the number of total neuromasts at 4 dpf (Sarrazin et al., 2010). As we observed earlier, RA signalling inhibition during late gastrulation and early segmentation stages reduced the speed of migratory primordium; we further checked how it affects the number of deposited neuromasts in late stages of development. We treated zebrafish embryos from 9-16 hpf with 
A

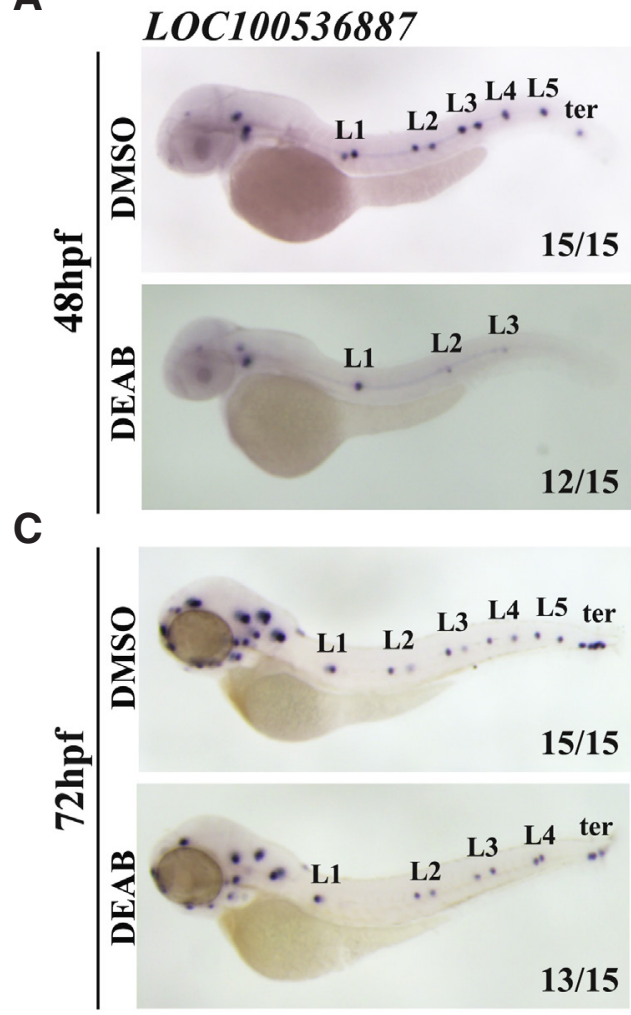

B
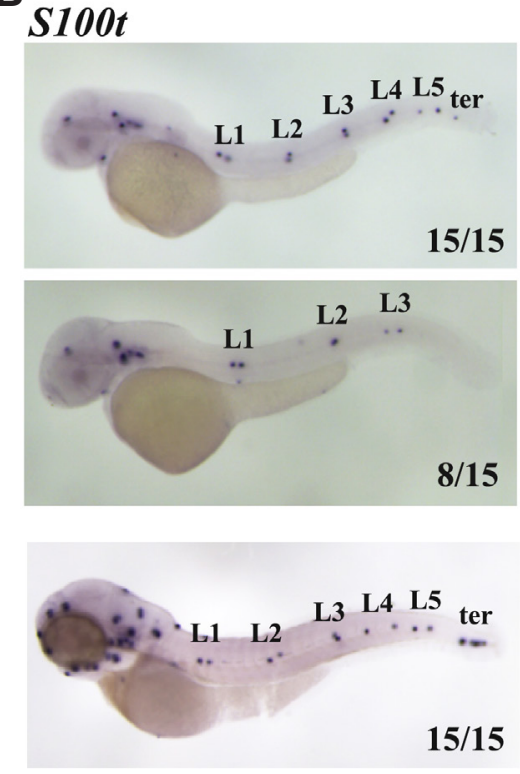

$15 / 15$

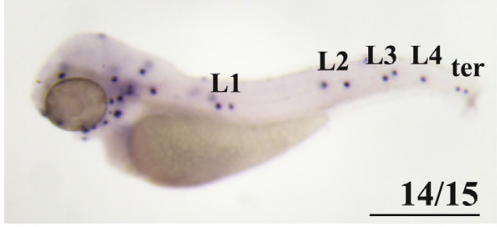

Fig. 6. Effect of DEAB on neuromast formation. (A) DEAB treated embryos showing reduced number of neuromasts compared with DMSO treated control embryos at 48 hpf. L1-L5 posterior neuromasts and terminal (ter) neuromasts were formed in control embryos whereas only L1-L3 were formed in DEAB treated embryos. Observations were done using LOC100536887 and S100t transcripts as neuromasts markers (B) Posterior neuromasts number were reduced in DEAB treated embryos at 72 hpf. Ratios at right corner of each image indicates number of embryos showing representative pattern. Scale bar $500 \mu \mathrm{m}$.

DEAB and fixed these at 48 and $72 \mathrm{hpf}$. LOC100536887 was used along with known neuromast marker S100t (Kraemer et al., 2008) to probe the neuromast deposition upon RA signalling inhibition. We found that $D E A B$ treated embryos showed a reduced number of neuromasts at $48 \mathrm{hpf}$. Only three neuromasts (L1-L3) were present in the trunk region in DEAB treated embryos, whereas the neuromast formation in trunk (L1-L5) and tail was normal in the vehicle (DMSO) control embryos (Fig. 6A). Four neuromasts (L1-L4), along with terminal neuromasts (ter), were observed in DEAB treated $72 \mathrm{hpf}$ embryos, whereas in vehicle control there were five (L1-L5), along with terminal neuromasts (Fig. 6B).

\section{Discussion}

In this study, we report identification of two genes that are annotated as uncharacterized in non-redundant databases because these genes or their homologs in other species have no known function. The expression pattern of a gene provides valuable clues to predicting its function. We have cloned these two genes and describe their mRNA expression pattern during zebrafish embryogenesis. We show that mRNA of uncharacterized protein LOC564095 precursor is expressed in migratory pLLP, and uncharacterized protein LOC100536887 is expressed in both anterior and posterior neuromasts. Thus, these genes may be involved in the development and function of zebrafish lateral line system. We further show that inhibition of RA synthesis by DEAB or citral treatment reduces their expression, suggesting that the expression of these genes may be regulated by RA signalling pathway. It is claimed that RA plays a major role in inducing posterior placode formation and primordium migration initiation during late gastrulation (Sarrazin et al., 2010; Nikaido et al., 2017). We targeted RA signalling pathway to check its effect on speed of primordium migration and neuromasts deposition using these two uncharacterized genes along with tgf $\beta 1 a$ and S100t as lateral line markers. We treated the embryos with DEAB at $9 \mathrm{hpf}$ before somitogenesis begins, and removed it at $16 \mathrm{hpf}$ prior to primordium migration. A late initiation of primordium migration was observed in DEAB treated embryos. The migratory speed of pLLP was also affected in $D E A B$ treated embryos, in which it was slow and irregular compared with DMSO vehicle control. The expression domain of migratory primordium was reduced in DEAB treated embryos as observed by the expression of both si:ch211-191i18.2 and tgf $\beta 1$ a gene (Fig. $5)$, which may indicate presence of smaller number of cells in the migratory primordium. There is a need to investigate whether inhibition of RA signalling reduces the number of migratory cells originating from the posterior lateral line placode. $60 \%$ of the length of migratory primordium is under the control of the Wnt signalling pathway (Dalle Nogare and Chitnis, 2017). As the primordium proliferates, the length of the leading Wnt system is reduced, gradually decreasing access of trailing end cells to Wnt dependent migratory factor. When access to this factor falls below a threshold level, the cells at the trailing end come under the influence of Fgf signalling pathway, halting migration and deposits as protoneuromasts. The rate at which primordium proliferates and the Wnt system shrinks determines the speed of migratory primordium (Aman et al., 2011; Valdivia et al., 2011; Dalle Nogare and Chitnis, 2017). We hypothesize that the reduced speed of pLLP migration in DEAB treated embryos could be a low rate of proliferation. The proliferation of migratory primordium regulate the process of neuromast deposition (Nechiporuk and Raible, 2008). The reduced number of neuromasts seen at $48 \mathrm{hpf}$ in DEAB treated embryos may also because of reduced cell proliferation, a hypothesis that requires further investigation.

\section{Materials and Methods}

\section{Bioinformatics and sequence analysis}

The NCBI's Conserved Domain Database was used to identify the domains present in uncharacterized protein LOC564095 precursor and uncharacterized protein LOC100536887. NCBI protein-BLAST was used to identify the homologous proteins present in other vertebrates. The PRA- 
LINE was used to analyse conserved amino acid residues. PRALINE uses homology-extended multiple alignment to enrich the information of each sequence present in the set. The output is visualized by means of different colour schemes to provide information about the position conservation of each residue (Simossis and Heringa, 2005). The MUSCLE multiple sequence alignment server, which is known for its speed and accuracy, was used to generate percentage identity matrix (Edgar, 2004).

\section{Zebrafish maintenance}

Tübingen, Albino and AB strains were used in all experiments. The animal work was approved by the Institutional Animal Ethics Committee. Embryos were raised in $\mathrm{E} 3$ media at $28.5^{\circ} \mathrm{C}$. Embryos were treated with $0.003 \%$ of 1-Phenyl 2 thiourea (PTU) in E3 media at $20 \mathrm{hpf}$ to inhibit pigmentation and fixed at different developmental stages in $4 \%$ paraformaldehyde (PFA).

\section{Gene cloning}

Zebrafish si:ch211-191118.2, LOC100536887, tgf $\beta 1 a$ and S100tgenes were amplified by PCR using cDNA prepared from $24 \mathrm{hpf}$ or $48 \mathrm{hpf} \mathrm{embryos.}$ Total RNAwas extracted using TRIzol reagent and cDNA was prepared using SuperScript ${ }^{\circledR}$ III (Thermo Fisher Scientific). The primers used for amplifying the genes were designed using http://primer3.ut.ee/ and are as follows: (si:ch211-191i18.2 forward 5'-AGCAGATGGACAGATAACCTGAA-3' and reverse 5'-CTGAATTCATACCAGCTATCGTG-3'; LOC100536887 forward 5'-ACCCTCTCAGGGGAACAGAT-3' and reverse 5'-CTGAGCTGGAACAAGCCGTA-3'; $\operatorname{tgf} \beta 1 a$ forward 5'-CCGAGATGAAGCGCAGTATTC-3' and reverse 5'-GTCAAGGATTGCGGGTACACA-3'; and S100t forward 5'-CCAGCAGTCATCTCACCTCG-3' and reverse 5'- AGACCATCGTACAAACTCACATCT-3'). Amplified products were cloned in PCR-Blunt II-TOPO vector (Invitrogen) and verified by sequencing.

\section{WISH Probe synthesis}

The above clones were used for probes synthesis. si:ch211-191i18.2, LOC10053688, tgf $\beta 1 a$ and S100t plasmids were linearized with Xhol and transcribed by SP6 RNA polymerase to synthesize anti-sense probe. Digoxygenin labelling mix (Roche 11277073910) or Fluorescein labelling mix (Roche 11685619910) were used to synthesize labelled RNA probe.

\section{Whole mount in situ hybridization}

WISH was performed on zebrafish embryos as described earlier (Thisse and Thisse, 2008). Briefly, embryos fixed in $4 \%$ PFA were dehydrated through a methanol series and stored at $-20^{\circ} \mathrm{C}$ in $100 \%$ methanol. The embryos were rehydrated in PBST (1X PBS with $0.1 \%$ Tween-20), permeabilized by proteinase $\mathrm{K}$ treatment $(10 \mu \mathrm{g} / \mathrm{ml}$ in 1 XPBST) and refixed with $4 \%$ PFA. These were kept in hybridization buffer $(50 \%$ deionized formamide, 5 X SSC, $50 \mu \mathrm{g} / \mathrm{ml}$ heparin, $0.5 \mathrm{mg} / \mathrm{ml}$ torula RNA, $9.2 \mathrm{mM}$ citric acid and $0.1 \%$ Tween-20) for $2-5$ hours at $65^{\circ} \mathrm{C}$. Later, the buffer was exchanged with hybridization buffer containing the probe. The next day, embryos were washed with formamide-SSC buffer and transferred into MABT (1X MAB with 0.1\% Tween-20) buffer. Embryos were kept in 10\% FBS (foetal bovine serum) and $2 \%$ blocking reagent (Roche 1109617600) in MABT for 2 hours at room temperature (RT) for blocking and then incubated with anti-digoxigenin $\mathrm{F}_{\mathrm{AB}}$ fragment coupled to alkaline phosphate (AP) (Roche 11093274910) or anti-fluorescein $F_{A B}$ fragment coupled to AP (Roche 11426338910) diluted in blocking buffer for overnight at $4^{\circ} \mathrm{C}$. On the $3^{\text {rd }}$ day, embryos were washed 8 times with MABT, twice with $\mathrm{pH} 9$ buffer (100 mM Tris- $\mathrm{HCl} \mathrm{pH} \mathrm{9.5,} 100 \mathrm{mM} \mathrm{NaCl}, 50 \mathrm{mM} \mathrm{MgCl} 2$ and $0.1 \%$ Tween-20) at RT and transferred into BM purple (Roche 11442074001) in dark until colour developed.

\section{Double in situ hybridization}

For Double in situ hybridization, embryos were incubated in hybridization buffer containing both digoxygenin and fluorescein labelled probes targeting two different mRNA. On the $4^{\text {th }}$ or $5^{\text {th }}$ day after the detection of first transcript with BM purple, embryos were washed with PBST followed by AP inactivation by treating them with methanol. Later, they were incubated in blocking buffer containing diluted antibody corresponding to the second probe. The next day, embryos were washed with MABT followed by $\mathrm{pH} 9$ buffer and incubated in dark with INT-BCIP (Roche 11681460001) for the development of second colour. The stained embryos were washed with PBST and transferred into 100\% glycerol for imaging. Images were taken using Leica MZ16 stereo microscope.

\section{Drug treatment}

$10 \mathrm{mM}$ stock solution of DEAB was prepared in DMSO, which was diluted in E3 media to a working concentration of $10-20 \mu \mathrm{M}$. $1 \mathrm{mM}$ stock solution of citral was prepared and diluted in E3 media to a working concentration of 1-2 $\mu \mathrm{M}$. Embryos were treated with DEAB, citral or DMSO (vehicle control) in dark at $28.5^{\circ} \mathrm{C}$. Embryos were washed three times with E3 media after treatment, and fixed at different stages of development as mentioned in results section.

\section{RT PCR}

Total RNA was extracted from pools of 10 embryos at different developmental stages of DMSO, DEAB and citral treated embryos using Direct-zol RNA miniprep kit (Zymo research). cDNA was synthesized using superscript IV (Invitrogen). The following reaction condition was set: $95^{\circ} \mathrm{C}, 5 \mathrm{~min} ;\left(95^{\circ} \mathrm{C}, 15 \mathrm{sec} ; 60^{\circ} \mathrm{C}, 30 \mathrm{sec}, 72^{\circ} \mathrm{C}, 30 \mathrm{sec}\right) \times 40$ cycles; $95^{\circ} \mathrm{C}$, $15 \mathrm{~min} ; 60^{\circ} \mathrm{C}, 1 \mathrm{~min} ; 95^{\circ} \mathrm{C}, 15 \mathrm{~min}$. Analysis was carried out in quadruplicates using $z E F 1 \alpha$ for normalization. For si:ch211-191i18.2 5'-TGCAGTCATTCAGGGGCTCA-3' forward and 5'-CACACTGATATCATCACACA-3' reverse, for LOC100536887 5'- GTGTGAGTAATAGTGCCACTG-3'forward and 5'- CCAAAATGTCAGTCGCATTC-3' reverse and for zEf1a 5'-ATCACCAAGGAAGTCAGCG-3'forward and 5'-ATCTTCCATCCCTTGAACCAG-3' reverse primers were used.

\section{Author contribution}

RKS conceived, supervised and provided resources for the project. SF carried out experiments and wrote the manuscript. RK and $A D$ carried out bioinformatics analysis that identified uncharacterized proteins, including two reported in this manuscript. All authors read and approved the manuscript.

\section{Acknowledgements}

This work was supported by extramural grants from SERB (EMR/2016/003780) and intramural funds from Institute of Life Sciences, Bhubaneswar, which is an autonomous institute of Department of Biotechnology (DBT), Ministry of Science and Technology, Govt. of India to RKS. SF is a recipient of DBT fellowship (DBT/2015/ILS/361). The authors thank Suryashikha Mohanty for technical assistance and managing the zebrafish facility.

\section{References}

AMAN A, PIOTROWSKI T (2008). Wnt/ $\beta$-Catenin and Fgf Signalling Control Collective Cell Migration by Restricting Chemokine Receptor Expression. Dev Cell 15: 749-761.

AMAN A, NGUYEN M, PIOTROWSKI T (2011). Wnt/ $\beta$-catenin dependent cell proliferation underlies segmented lateral line morphogenesis. Dev Biol 349: 470-482.

ANDERMANN P, UNGOS J, RAIBLE DW (2002). Neurogenin1 Defines Zebrafish Cranial Sensory Ganglia Precursors. Dev Biol 251: 45-58.

BLECKMANN H, ZELICK R (2009). Lateral line system of fish. Integr Zool 4: 13-25.

BRENT MR (2005). Genome annotation past, present, and future: How to define an ORF at each locus. Genome Res 15: 1777-1786.

CHITNIS AB, DALLE NOGARE D, MATSUDA M (2012). Building the posterior latera line system in zebrafish. Dev Neurobiol 72: 234-255.

COOMBS S, VAN NETTEN S (2005). The Hydrodynamics and Structural Mechanics of the Lateral Line System. In Fish Biomechanics (Eds Shadwick RE and Lauder GV). Elsevier, Inc. pp. 103-139.

DALLE NOGARE D, CHITNIS AB (2017). A framework for understanding morphogenesis and migration of the zebrafish posterior Lateral Line primordium. Mech 
Dev 148: 69-78.

DAMBLY-CHAUDIĖRE C, CUBEDO N, GHYSEN A (2007). Control of cell migration in the development of the posterior lateral line: Antagonistic interactions between the chemokine receptors CXCR4 and CXCR7/RDC1. BMC Dev Biol 7: 1-14.

DAVID NB, SAPEDE D, SAINT-ETIENNE L, THISSE C, THISSE B, DAMBLYCHAUDIERE C, ROSA FM, GHYSEN A (2002). Molecular basis of cell migration in the fish lateral line: Role of the chemokine receptor CXCR4 and of its ligand, SDF1. Proc Natl Acad Sci 99: 16297-16302.

EDGAR RC (2004). MUSCLE: Multiple sequence alignment with high accuracy and high throughput. Nucleic Acids Res 32: 1792-1797.

GHYSEN A, DAMBLY-CHAUDIĖRE C (2004). Development of the zebrafish lateral line. Curr Opin Neurobiol 14: 67-73.

GOMPELN, CUBEDO N, THISSE C, THISSE B, DAMBLY-CHAUDIĖRE C, GHYSEN A (2001). Pattern formation in the lateral line of zebrafish. Mech Dev 105: 69-77.

HAAS P, GILMOURD (2006). Chemokine Signalling Mediates Self-Organizing Tissue Migration in the Zebrafish Lateral Line. Dev Cell 10: 673-680.

HAVA D, FORSTER U, MATSUDA M, CUI S, LINK BA, EICHHORST J, WIESNER B, CHITNIS A, ABDELILAH-SEYFRIED S (2009). Apical membrane maturation and cellular rosette formation during morphogenesis of the zebrafish lateral line. $J$ Cell Sci 122: 687-695.

HAWKINST, KIHARAD (2007). FUNCTION PREDICTION OF UNCHARACTERIZED PROTEINS. J Bioinform Comput Biol 5: 1-30.

IJAQ J, CHANDRASEKHARAN M, PODDAR R, BETHI N, SUNDARARAJAN VS (2015). Annotation and curation of uncharacterized proteins- challenges. Front Genet 6: 1-7.

KORNBLUM HI, CORWIN JT, TREVARROW B (1990). Selective labeling of sensory hair cells and neurons in auditory, vestibular, and lateral line systems by a monoclonal antibody. J Comp Neuro/ 301: 162-170.

KRAEMER AM, SARAIVA LR, KORSCHING SI (2008). Structural and functional diversification in the teleost $\mathrm{S} 100$ family of calcium-binding proteins. BMC Evol Biol 8: 1-23.

LECAUDEY V, CAKAN-AKDOGAN G, NORTON WHJ, GILMOURD (2008). Dynamic Fgf signalling couples morphogenesis and migration in the zebrafish lateral line primordium. Development 135: 2695-2705.

LESPINET O (2002). The Role of Lineage-Specific Gene Family Expansion in the Evolution of Eukaryotes. Genome Res 12: 1048-1059.

MATSUDA M, CHITNIS AB (2010). Atoh1a expression must be restricted by Notch signalling for effective morphogenesis of the posterior lateral line primordium in zebrafish. Development 137: 3477-3487.

METCALFE WK (1985). Sensory neuron growth cones comigrate with posterior lateral line primordial cells in zebrafish. J Comp Neurol 238: 218-224.

NECHIPORUK A, RAIBLE DW (2008). FGF-Dependent Mechanosensory Organ Patterning in Zebrafish. Science 320: 1774-1777.

NIKAIDO M, NAVAJAS ACEDO J, HATTA K, PIOTROWSKI T (2017). Retinoic acid is required and Fgf, Wnt, and Bmp signalling inhibit posterior lateral line placode induction in zebrafish. Dev Biol 431: 215-225.

NUÑEZ VA, SARRAZIN AF, CUBEDO N, ALLENDE ML, DAMBLY-CHAUDIĖRE C GHYSEN A (2009). Postembryonic development of the posterior lateral line in the zebrafish. Evol Dev 11: 391-404.

SARRAZIN AF, NUNEZ VA, SAPEDE D, TASSIN V, DAMBLY-CHAUDIERE C, GHYSEN A (2010). Origin and Early Development of the Posterior Lateral Line System of Zebrafish. J Neurosci 30: 8234-8244.

SIMOSSIS VA, HERINGAJ (2005). PRALINE: a multiple sequence alignment toolbox that integrates homology-extended and secondary structure information. Nucleic Acids Res 33: W289-W294.

THISSE C, THISSE B (2008). High-resolution in situ hybridization to whole-mount zebrafish embryos. Nat Protoc 3: 59-69.

VALDIVIALE, YOUNG RM, HAWKINSTA, STICKNEYHL, CAVODEASSIF, SCHWARZ Q, PULLIN LM, VILLEGAS R, MORO E, ARGENTON F, ALLENDE ML, WILSON SW (2011). Lef1-dependent Wnt/-catenin signalling drives the proliferative engine that maintains tissue homeostasis during lateral line development. Development 138: 3931-3941.

XING C, GONG B, XUE Y, HAN Y, WANG Y, MENG A, JIA S (2015). TGF $\beta 1$ a regulates zebrafish posterior lateral line formation via Smad5 mediated pathway. Mol Cell Biol 7: 48-61. 


\section{Further Related Reading, published previously in the Int. J. Dev. Biol.}

Expression of two uncharacterized protein coding genes in zebrafish lateral line system Sana Fatma, Ravindra Kumar, Anshuman Dixit and Rajeeb K. Swain

Int. J. Dev. Biol. (2021) 52: 66-66

https://doi.org/10.1387/ijdb.210066rs

Development vs. behavior: a role for neural adaptation in evolution?

Alain Ghysen and Christine Dambly-Chaudière

Int. J. Dev. Biol. (2016) 60: 77-84

https://doi.org/10.1387/ijdb.160097ag

Calnexin is required for zebrafish posterior lateral line development

I-Chen Hung, Bor-Wei Cherng, Wen-Ming Hsu and Shyh-Jye Lee

Int. J. Dev. Biol. (2013) 57: 427-438

https://doi.org/10.1387/ijdb.120166sl

Trans-2-phenylcyclopropylamine regulates zebrafish lateral line neuromast development mediated by depression of LSD1 activity Yingzi He, Huiqian Yu, Shan Sun, Yunfeng Wang, Liman Liu, Zhengyi Chenand Huawei Li Int. J. Dev. Biol. (2013) 57: 365-373

https://doi.org/10.1387/ijdb.120227hl

Development of the posterior lateral line system in Thunnus thynnus, the atlantic bluefin tuna, and in its close relative Sarda sarda

Alain Ghysen, Kevin Schuster, Denis Coves, Fernando de la Gandara, Nikos Papandroulakis and Aurelio Ortega

Int. J. Dev. Biol. (2010) 54: 1317-1322

https://doi.org/10.1387/ijdb.103102ag

Retinoic acid repatterns axolotl lateral line receptors

Melissa A Gibbs and R Glenn Northcutt

Int. J. Dev. Biol. (2004) 48: 63-66

http://www.intjdevbiol.com/web/paper/15005576

The cytoskeletal effector XPAK1 is expressed during both ear and lateral line development in Xenopus

$\mathrm{N}$ Islam, L Poitras and T Moss

Int. J. Dev. Biol. (2000) 44: 245-248

http://www.intjdevbiol.com/web/paper/10794083

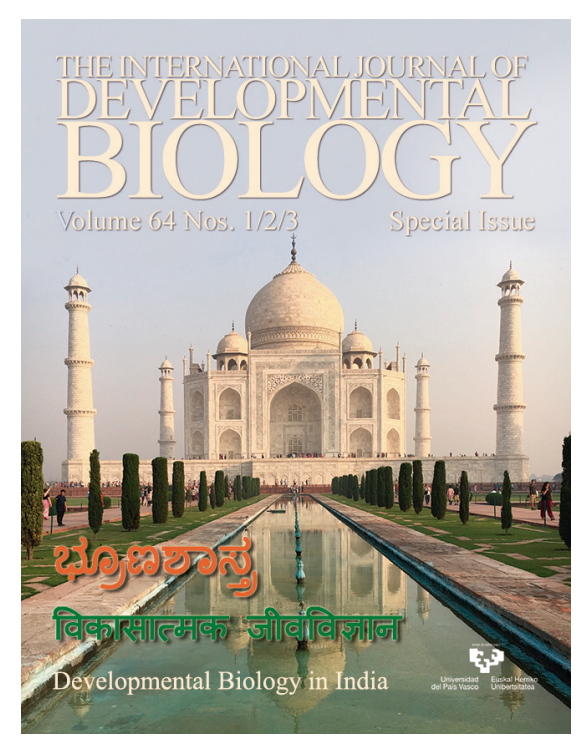

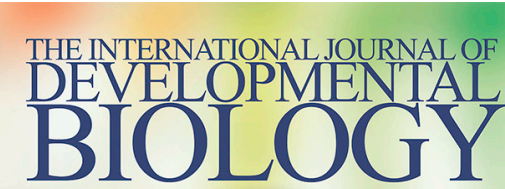

Volume 65 Nos. $1 / 2 / 3$ Special Issue

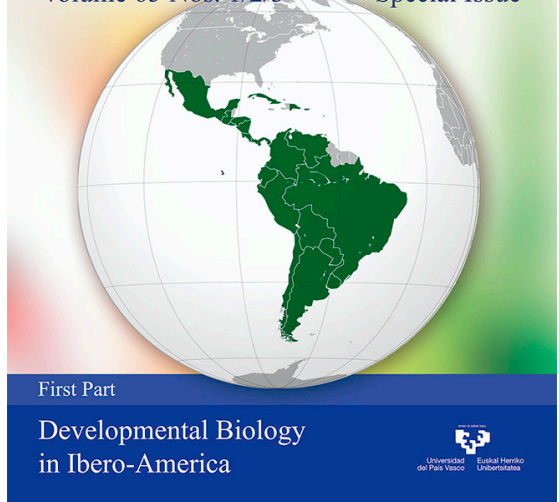

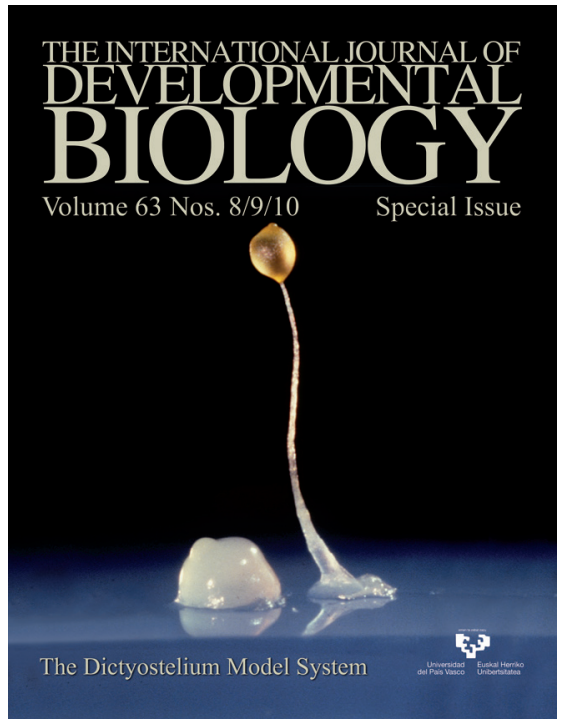

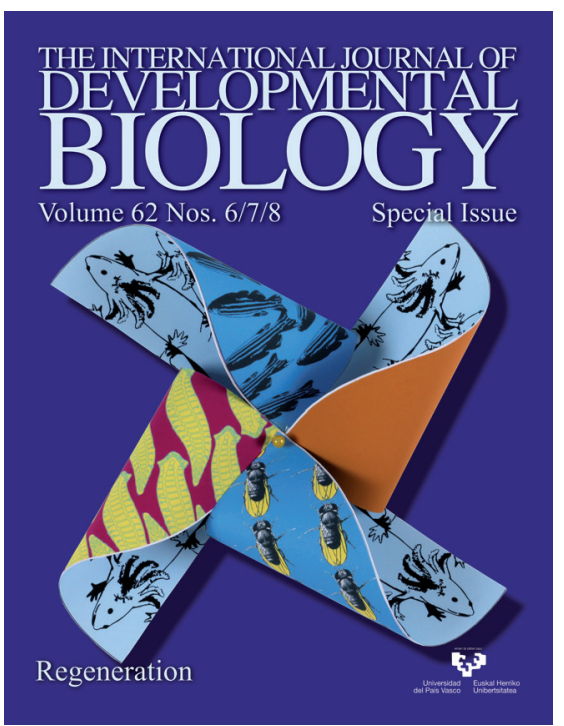

